\title{
Terrestrial Ozone Depletion Due to a Milky Way Gamma-Ray Burst
}

Brian C. Thomas ${ }^{1}$, Charles H. Jackman ${ }^{2}$, Adrian L. Melott ${ }^{1}$, Claude M. Laird ${ }^{1,4}$, Richard S. Stolarski ${ }^{2}$, Neil Gehrels ${ }^{3}$, John K. Cannizzo ${ }^{3}$, and Daniel P. Hogan ${ }^{1}$

\begin{abstract}
Based on cosmological rates, it is probable that at least once in the last Gy the Earth has been irradiated by a gamma-ray burst in our Galaxy from within 2 kpc. Using a two-dimensional atmospheric model we have computed the effects upon the Earth's atmosphere of one such burst. A ten second burst delivering $100 \mathrm{~kJ} / \mathrm{m}^{2}$ to the Earth results in globally averaged ozone depletion of $35 \%$, with depletion reaching $55 \%$ at some latitudes. Significant global depletion persists for over 5 years after the burst. This depletion would have dramatic implications for life since a $50 \%$ decrease in ozone column density results in approximately three times the normal UVB flux. Widespread extinctions are likely, based on extrapolation from UVB sensitivity of modern organisms.
\end{abstract}

Subject headings: astrobiology - gamma rays: bursts

\section{Introduction}

Camma-ray bursts (GRB) within our galaxy have been suggested as a possible threat to life on Earth (Thorsett 1995; Scalo \& Wheeler 2002; Dar \& De Rujula 2002; Melott et al.

\footnotetext{
${ }^{1}$ University of Kansas, Department of Physics and Astronomy 1251 Wescoe Hall Dr, Room 1082

Lawrence, KS 66045-7582; bthomas@ku.edu

${ }^{2}$ Laboratory for Atmospheres

NASA Goddard Space Flight Center

Code 916

Greenbelt, MD 20771

${ }^{3}$ Laboratory for High Energy Astrophysics NASA Goddard Space Flight Center

Code 661

Greenbelt, MD 20771

${ }^{4}$ Also at Haskell Indian Nations University
} 
2004). Some effects similar to that due to a nearby supernova (SN) are expected, especially depletion of stratospheric ozone due to ionization caused by incident gamma radiation. GRB are rarer than $\mathrm{SNe}$, but their greater energy output results in a larger region of influence and hence they may pose a greater threat. Results presented in Melott et al. (2004) indicate that a GRB impact is likely within the last Gy. Such a GRB is expected to have dramatic effects on stratospheric ozone, leading to detrimental effects on life through increases in solar ultraviolet (UV) radiation which is strongly absorbed by ozone.

In order to gain more detailed and accurate insight into these expected effects we have performed computations using the Goddard Space Flight Center (GSFC) two-dimensional atmospheric model. This model has been used previously to investigate the atmospheric effects of SN (Gehrels et al. 2003) and we build on that work.

\section{Methods}

We take as "typical" a GRB with power $5 \times 10^{44} \mathrm{~W}$ (isotropic equivalent) and duration 10 $\mathrm{s}$, whose gamma-ray spectrum is described by the Band spectrum (Band et al. 1993). These assumptions are drawn from observations and are not dependent upon beaming angle. We have determined the depletion of ozone for such a GRB beamed at the Earth from a distance of $2 \mathrm{kpc}$, delivering to the Earth a total fluence of $100 \mathrm{~kJ} / \mathrm{m}^{2}$. This distance corresponds to that of a probable nearest "typical" GRB in the last Gy, based on conservative assumptions (Melott et al. 2004).

The prompt effect of this burst at the Earth's surface is a "flash" of UVB radiation with power $\approx 20 \mathrm{~W} / \mathrm{m}^{2}$ (Smith et al. 2003). This is about seven times the intensity at the Earth's surface on a bright, sunny day, but is brief and so is not likely to have a major effect on life. Longer term effects include ozone depletion and the resulting increase in solar UVB flux, which we begin to explore here.

We have not included the effects of any ultra high energy $\left(>10^{18} \mathrm{eV}\right)$ cosmic rays from a GRB (Dermer \& Atoyan 2004), due to uncertainty as to whether and at what energies GRB may produce such particles. Lower energy cosmic rays which may be produced are negligible at our assumed distances due to deflection by the galactic magnetic field. It is estimated (Dermer 2004) that inclusion of ultra high energy cosmic rays would add about $30 \%$ to the ozone-destroying energy budget. We would also expect production of some radioisotopes from cosmic ray spallation off of atmospheric constituents, as well as a terrestrial burst of muons. 


\subsection{Atmospheric Model}

The GSFC 2D model is described in Douglass, Jackman, \& Stolarski (1989); Jackman et al. (1990); and Considine, Douglass, \& Jackman (1994). The model's two dimensions are latitude and altitude (ranging up to about $116 \mathrm{~km}$ ). The latitude range is divided into 18 equal bands and extends from pole to pole. The altitude range includes 58 evenly spaced logarithmic pressure levels (approximately $2 \mathrm{~km}$ spacing). A lookup table is used for computation of photolytic source term, used in calculations of photodissociation rates of atmospheric constituents by sunlight (Jackman et al. 1996). Winds and small scale mixing are included as described in Fleming et al. (1999). For this study, we have removed anthropogenic compounds such as CFCs.

We have employed two versions of the atmospheric model. One is intended for long term runs (many years) and includes all transport mechanisms (e.g., winds and diffusion). This version has a time step of one day and computes daily averaged constituent values. The second version is used for short term runs (a few days) and calculates constituent values throughout the day and night, but does not include transport. Previously, this version has been used with a time step of 225 seconds (Jackman et al. 2001). In the current study we have used a time step of one second in order to allow for inputting our GRB gamma radiation over several time steps.

Gamma-rays are introduced in the model in a manner similar to that described in Gehrels et al. (2003). In that study, gamma-rays were included using the spectrum of SN $1987 \mathrm{~A}$. In the present study, the gamma-ray differential photon count spectrum used is that of Band et al. (1993), which consists of two smoothly connected power laws. We use the following values for the break energy and power law indices, respectively: $E_{0}=187.5 \mathrm{keV}$, $\alpha=-0.8, \beta=-2.3$ (Preece et al. 2000). The total received energy is scaled to our desired value (in this study, corresponding to a fluence of $100 \mathrm{~kJ} / \mathrm{m}^{2}$ ) The total photon flux in each of 66 evenly spaced logarithmic energy bins, ranging $0.001 \leqq E \leqq 10 \mathrm{MeV}$, is obtained by integrating the Band spectrum for each bin.

\subsection{Simulations}

All simulation runs used for analysis were begun with initial conditions obtained from a long-term (roughly 40 years) run intended to bring the model to equilibrium. Constituent values from this run are read in by the 1 s time step version of the model which runs for 7 days (beginning at noon), either with or without input of gamma-rays. Runs including gamma radiation input the burst as a step function at noon on day 4 , with duration $10 \mathrm{~s}$. In the 
current study, the burst is input in late March (near the spring equinox) with incident angle 0 degrees (equatorial). (Forthcoming studies will investigate the effects of varying intensity, incidence angle, and times of year at which the burst occurs.) Day 4 is the middle of the short term run, which allows for "warm up" in the model and insures that relevant chemistry is accurately computed over many time steps after the burst. Constituent values from this type of run are then read in by the 1 day time step version which is run for 20 years in order to investigate long term effects and determine how long the atmosphere takes to return to equilibrium, pre-burst conditions.

Analysis is then performed by comparing such a combined base-short-long run without gamma-ray input to such a run with the burst included. Ozone depletion and other effects are computed by comparing these two runs.

\section{Results}

Stratospheric ozone is lost through several catalytic reactions involving oxygen-, nitrogen, hydrogen-, chlorine-, and bromine-containing gases. The constituents in the stratosphere are generally grouped into "families" such as $\mathrm{O}_{\mathrm{x}}\left(\mathrm{O}_{3}, \mathrm{O}, \mathrm{O}\left({ }^{1} \mathrm{D}\right)\right), \mathrm{NO}_{\mathrm{y}}\left(\mathrm{N}, \mathrm{NO}, \mathrm{NO}_{2}, \mathrm{NO}_{3}\right.$, $\mathrm{N}_{2} \mathrm{O}_{5}, \mathrm{HNO}_{3}, \mathrm{HO}_{2} \mathrm{NO}_{2}, \mathrm{ClONO}_{2}, \mathrm{BrONO}_{2}$ ), $\mathrm{HO}_{\mathrm{x}}\left(\mathrm{H}, \mathrm{OH}, \mathrm{HO}_{2}\right.$ ), Cly (chlorine-containing inorganic molecules), and Bry (bromine-containing inorganic molecules), which allow for efficient computation of the chemistry and transport effects. We assumed that there were no human-made sources for any of these families, as such large-scale anthropogenic perturbations have been present only over a relatively brief period of time $(\approx$ decades$)$. Our model simulations are thus focused on a period of geologic time wherein the ozone layer was substantial, but the human influence was negligible.

In the case of a large input of gamma rays to the atmosphere $\mathrm{NO}_{\mathrm{y}}$ compounds (most importantly $\mathrm{NO}$ and $\mathrm{NO}_{2}$ ) are created through dissociation of $\mathrm{N}_{2}$ which then reacts with $\mathrm{O}_{2}$ to generate $\mathrm{NO}$. Subsequent reactions create $\mathrm{NO}_{2}$ and other compounds. Together, these react catalytically to deplete $\mathrm{O}_{3}$ through the cycle

$$
\begin{array}{r}
\mathrm{NO}+\mathrm{O}_{3} \rightarrow \mathrm{NO}_{2}+\mathrm{O}_{2} \\
\mathrm{NO}_{2}+\mathrm{O} \rightarrow \mathrm{NO}+\mathrm{O}_{2} \\
\text { net : } \mathrm{O}_{3}+\mathrm{O} \rightarrow \mathrm{O}_{2}+\mathrm{O}_{2}
\end{array}
$$

Note that $\mathrm{NO}$ is not consumed in this cycle and the net result is the destruction of $\mathrm{O}_{3}$ and production of $\mathrm{O}_{2}$. Other reactions can complicate this cycle, such as destruction of $\mathrm{NO}$ by reaction with $\mathrm{N}$; production of $\mathrm{O}_{3}$ through reactions of $\mathrm{NO}$ with $\mathrm{HO}_{2}$; and interference of 
$\mathrm{NO}_{\mathrm{y}}$ with other families (chlorine-, bromine-, and hydrogen-containing constituents) which reduces the ozone depletion from these families. Some uncertainties in the atmospheric model's treatment of this cycle are discussed in Section 4.

The primary results of our simulations are increases in $\mathrm{NO}_{\mathrm{y}}$ and decreases in $\mathrm{O}_{3}$. Ozone column densities can then be used to calculate the resulting UVB flux at the Earth's surface and from this information biological effects can be estimated. UVB is particularly damaging to organisms because DNA absorbs strongly in this wavelength region and is damaged.

Results of our modeling are shown in Figures 1 and 2. We have modeled the effects of $100 \mathrm{~kJ} / \mathrm{m}^{2}$ total received gamma-ray fluence, input as described in Sect. 2.2. This corresponds to our "typical" GRB located at about $2 \mathrm{kpc}$. Figure 1 shows the percent difference (between a run with gamma-ray input and one without) in vertical column density of $\mathrm{O}_{3}$. The burst is input at time 0 and immediate depletion of ozone is evident. Due to their qualitative similarity, we have chosen not to plot here changes in $\mathrm{NO}_{\mathrm{y}}$. Maximum increase in $\mathrm{NO}_{\mathrm{y}}$ is largely coincident with maximum decrease in $\mathrm{O}_{3}$. The maximum increase in $\mathrm{NO}_{\mathrm{y}}$ is approximately 30 -fold for this case.

Figure 2 shows the vertical column density of $\mathrm{O}_{3}$ at each latitude over time. Again, the burst is input at time 0 . Included are scales in both Dobson units (the usual unit of ozone column density) and $10^{18} \mathrm{~cm}^{-2}$. A Dobson unit describes the thickness of a column of ozone at standard temperature and pressure and is defined as $1 \mathrm{DU}=0.01 \mathrm{~mm}$ thickness (or, $1 \mathrm{DU}=2.69 \times 10^{18} \mathrm{~cm}^{-2}$ ).

Several features in these plots are worth noting. First, as is seen in Fig. 1 and 2, depletion of ozone is initially greatest at the equator (where the incident flux is highest), becoming greatest toward the poles within a year or so. Larger ozone depletions at the poles are primarily due to the long lifetime of the enhanced $\mathrm{NO}_{\mathrm{y}}$ in the polar stratosphere. Fig. 1 gives a somewhat exaggerated impression of the effect of depletion at the poles, since ozone is initially high there. The enhanced $\mathrm{NO}_{y}$, including $\mathrm{HNO}_{3}$, will lead to an enhancement of nitric acid trihydrate (NAT) polar stratospheric clouds (PSCs). These NAT PSCs facilitate heterogeneous reactions that result in greater ozone depletion by halogen (chlorine and bromine) constituents. This is especially true in the south polar region where a stronger polar vortex with colder stratospheric temperatures is in place during the winter. This contributes to an asymmetry which would be peculiar to the present-day configuration of continents. An additional effect which contributes to the polar asymmetry is the time of year at which the burst occurs, since ozone concentrations at the poles exhibit large seasonal variations.

Note, however, that around 5-6 months after the burst there is a short term production of 
ozone toward the south pole. Production occurs at the end of south polar night when a lack of photolysis has caused accumulation of $\mathrm{NO}_{\mathrm{y}}$ constituents which are suddenly photolysized as the sun rises, producing $\mathrm{O}$ which may then react with $\mathrm{O}_{2}$ to form $\mathrm{O}_{3}$. This production is fairly short lived as the $\mathrm{O}$ is consumed by various reactions and remaining $\mathrm{NO}_{\mathrm{y}}$ compounds continue to destroy $\mathrm{O}_{3}$.

Overall, globally averaged ozone depletion reaches about 35\% (at the start of the longterm run) and a maximum depletion of about $55 \%$ occurs first at the equator immediately after the burst, and then again about 15 months after the burst, in the southern hemisphere. Significant global depletion (10\% or more) lasts for over 5 years after the burst.

Figure 3 shows DNA damage estimated by convolving the daily average UVB flux with a biological weighting function (Setlow 1974; Smith et al. 1980). We have normalized the plot by dividing the damage by the annual global average damage in the absence of a GRB. Greater DNA damage probability is evident at low latitudes. This is due to combination of the $\mathrm{O}_{3}$ depletion effects with the sun incidence angle, length of day, etc. One might think that this damage would be countered by a greater evolved UVB-resistance in organisms at low latitudes. However, at least for modern phytoplankton, there is no evidence that temperate zone phytoplankton are any more UVB-resistant than Antarctic plankton (Prezelin 2004). Thus, at least for this burst geometry, one might predict that greater ecological damage and extinction would be likely near the equator. Of course, early in the Earth's history $\mathrm{O}_{2}$, and hence $\mathrm{O}_{3}$, levels were lower than today (Thomas et al. (2004) and references therein) and life may have been more UVB-resistant, lessening this impact.

\section{Uncertainties}

Our ionization profiles are computed-using simple energy-dependent attenuation coefficients, instead of a full radiative transfer calculation. This technique is implemented following Gehrels et al. (2003) with the primary modification being the functional form of the spectrum. That study found good agreement between their calculations and a full radiative transfer model. Similarly, we find that ionization due to the gamma-ray input peaks around $30 \mathrm{~km}$ elevation, which is in agreement with Gehrels et al. and Smith et al. (2003).

A discussion of some uncertainties in the production of $\mathrm{NO}_{\mathrm{y}}$ compounds is presented in Melott et al. (2004). In particular, reactions involving exited state nitrogen atoms, $N\left({ }^{2} \mathrm{D}\right)$, are not included in the atmospheric model. Comparison with simplified, off-line computations using various ratios of $N\left({ }^{2} \mathrm{D}\right) / \mathrm{N}\left({ }^{4} \mathrm{~S}\right)$ indicate that for our present case the effect on $\mathrm{NO}$ production of including excited state $\mathrm{N}$ atoms is small. Though results depend on the 
assumed temperature at which the reactions occur, for a reasonable range of temperatures $(230-270 \mathrm{~K})$, there is little variation of NO production with increased concentrations of excited state $\mathrm{N}$ atoms, and there is generally less than a factor of two difference between the off-line computations and model results.

The GSFC two-dimensional atmospheric model is empirically based and its dynamics are not coupled to the significantly changing constituent levels and accompanying heating. This fact introduces some uncertainty in the transport of constituents.

\section{Discussion}

A significant result of our modeling is that even for a short duration input of radiation, atmospheric effects are large and long-lived. Expectations based on supernova studies indicated that a short duration input might not have such large effects (Gehrels et al. 2003).

Appearance of features such as the localized production of $\mathrm{O}_{3}$ highlights the need for detailed modeling of the effects of a GRB on the Earth's atmosphere, as complexities and feedbacks in the chemical processes can be important and simple scaling may not prove reliable.

Melott et al. (2004) summarizes studies of UVB sensitivity of various organisms (see also Cockell (1999)). About $90 \%$ of UVB is presently absorbed by atmospheric ozone. Due to the sensitivity of DNA to this radiation, increases of only 10-30\% can have lethal effects on many organisms, especially phytoplankton, the base of the food chain. Ozone depletions in the range of $50 \%$, as seen here, lead to roughly three times more UVB at the surface, which is clearly a possible candidate for causing mass extinctions.

B.C.T. acknowledges support from Neil Gehrels at NASA Goddard Space Flight Center. B.C.T., A.L.M. and C.L. acknowledge support from NASA grant NNG04GM41G. D.P.H. acknowledges support from an Undergraduate Research Award through the Honors Program at the University of Kansas. B.C.T. and A.L.M. acknowledge supercomputing support from the National Center for Supercomputing Applications. A.L.M. wishes to thank J. Scalo, D. Smith, and J.C. Wheeler for useful conversations.

\section{REFERENCES}

Band, D., et al. 1993, ApJ, 413, 281 
Cockell, C. 1999, Paleobiology, 25, 212

Considine, D.B., Douglass, A.R., \& Jackman, C.H. 1994, J. Geophys. Res., 99, 18879

Dar, A. \& De Rujula, A. 2002, in Astrophysics and Gamma Ray Physics in Space, eds. A. Morselli \& P. Picozza (Frascati Physics Series Vol. XXIV), 513

Dermer, C. \& Atoyan, A. 2004, New Astronomy Reviews, 48, 453

Dermer, C. 2004, personal communication

Douglass, A.R., Jackman, C.H., \& Stolarski, R.S. 1989, J. Geophys. Res., 94, 9862

Fleming, E.L., Jackman, C.H., Stolarski, R.S., \& Considine, D.B. 1999, J. Geophys. Res., 104, 23911

Gehrels, N., Laird, C.M., Jackman, C.H., Cannizzo, J.K., Mattson, B.J., \& Chen, W. 2003, ApJ, 585, 1169

Jackman, C.H., McPeters, R.D., Labow, G.J., Fleming, E.L., Praderas, C.J., \& Russell, J.M. 2001, Geophys. Res. Lett., 28, 2883

Jackman, C.H., Douglass, A.R., Rood, R.B., McPeters, R.D., \& Meade, P.E. 1990, J. Geophys. Res., 95, 7417

Jackman, C.H., Fleming, E.L., Chandra, S., Considine, D.B., \& Rosenfield, J.E. 1996, J. Geophys. Res., 101, 28753

Melott, A.L., Lieberman, B.S., Laird, C.M., Martin, L.D., Medvedev, M.V., Thomas, B.C., Cannizzo, J.K, Gehrels, N., \& Jackman, C.H. 2004, Int. J. Astrobiology, 3, 55

Preece, R.D., et al. 2000, ApJS, 126, 19

Prezelin, B. 2004, personal communication

Scalo, J., \& Wheeler, J.C. 2002, ApJ, 566, 723

Setlow, R.B. 1974, Pro. Nat. Acad. Sci. USA, 71, 3363

Smith, D.S., Scalo, J., \& Wheeler, J.C. 2003, Icarus, 171, 229

Smith, R.C., Baker, K.S., Holm-Hansen, O., \& Olson, R. 1980, Photochem. Photobiol., 31, 585 
Thomas, B.C., Melott, A.L., Martin, L.D., Jackman, C.H. 2004, Astrobiology, submitted (astro-ph/0410555)

Thorsett, S.E. 1995, ApJ, 444, L53

This preprint was prepared with the AAS IATEX macros v5.2. 


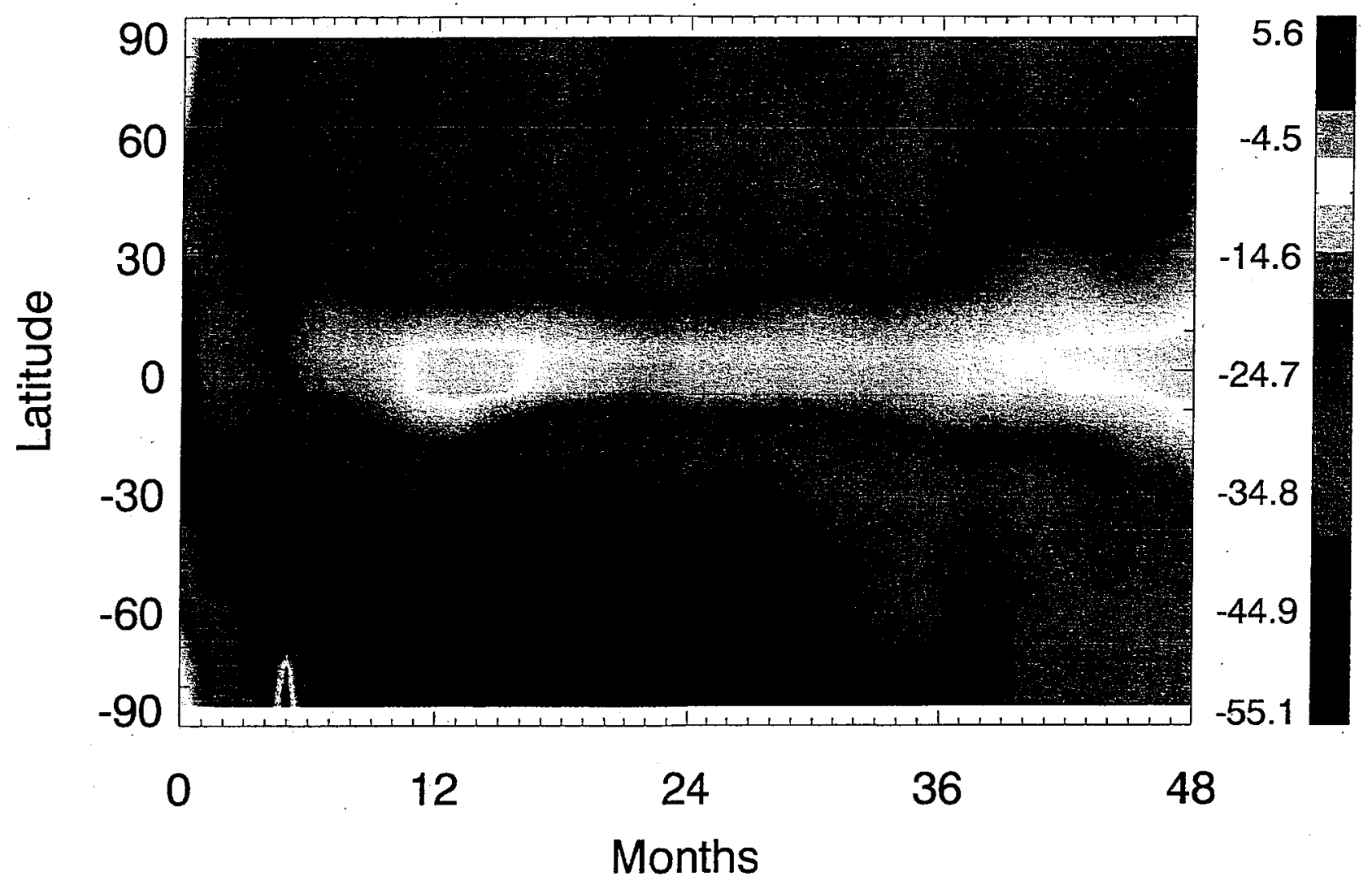

Fig. 1.- Percent change in column density of ozone (comparing runs with and without burst). 


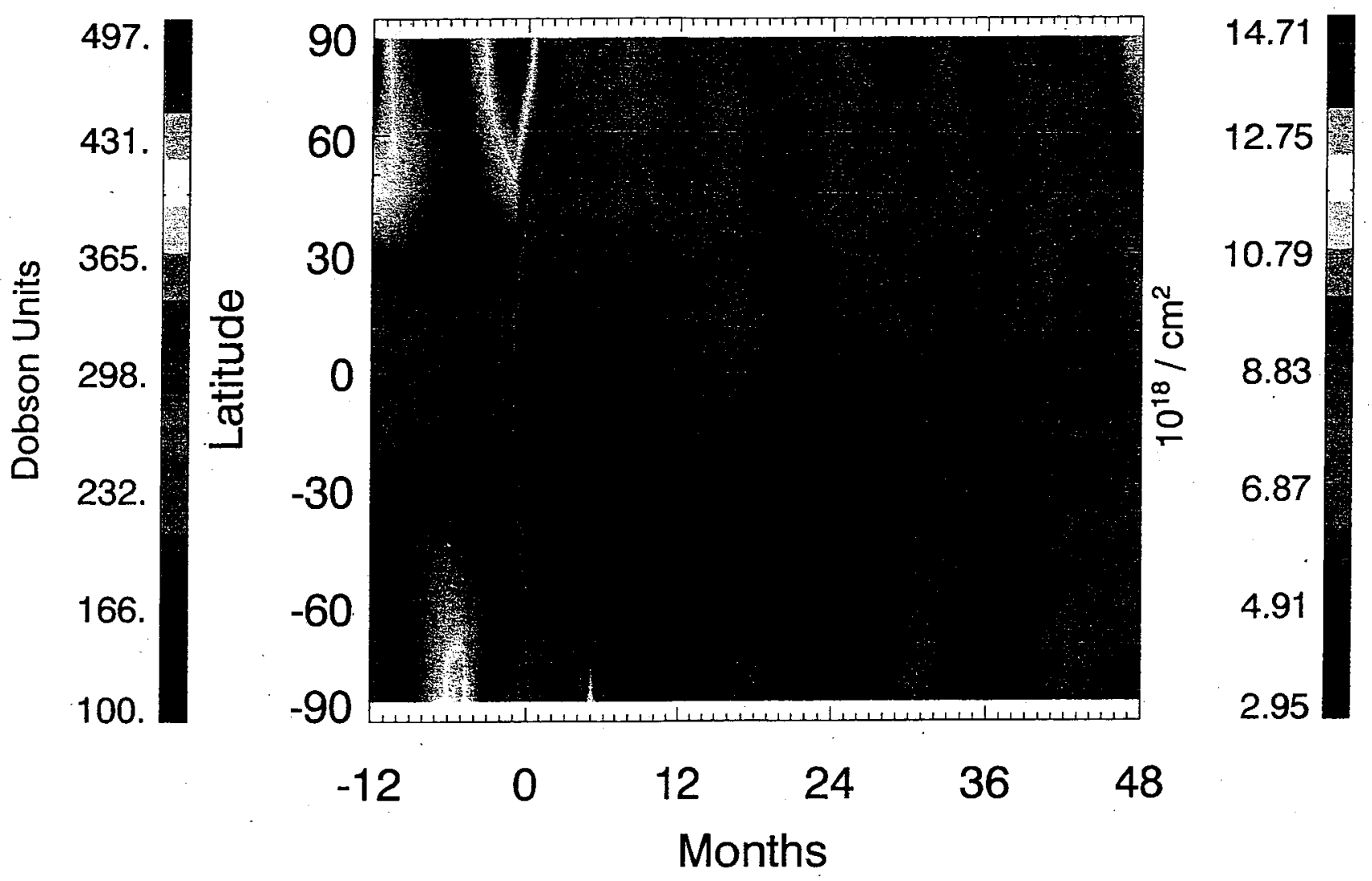

Fig. 2.- Column density of $\mathrm{O}_{3}$ with scales for both Dobson units (left) and $10^{18} \mathrm{~cm}^{-2}$ (right). (Plotted from one year before the burst to four years after.) 


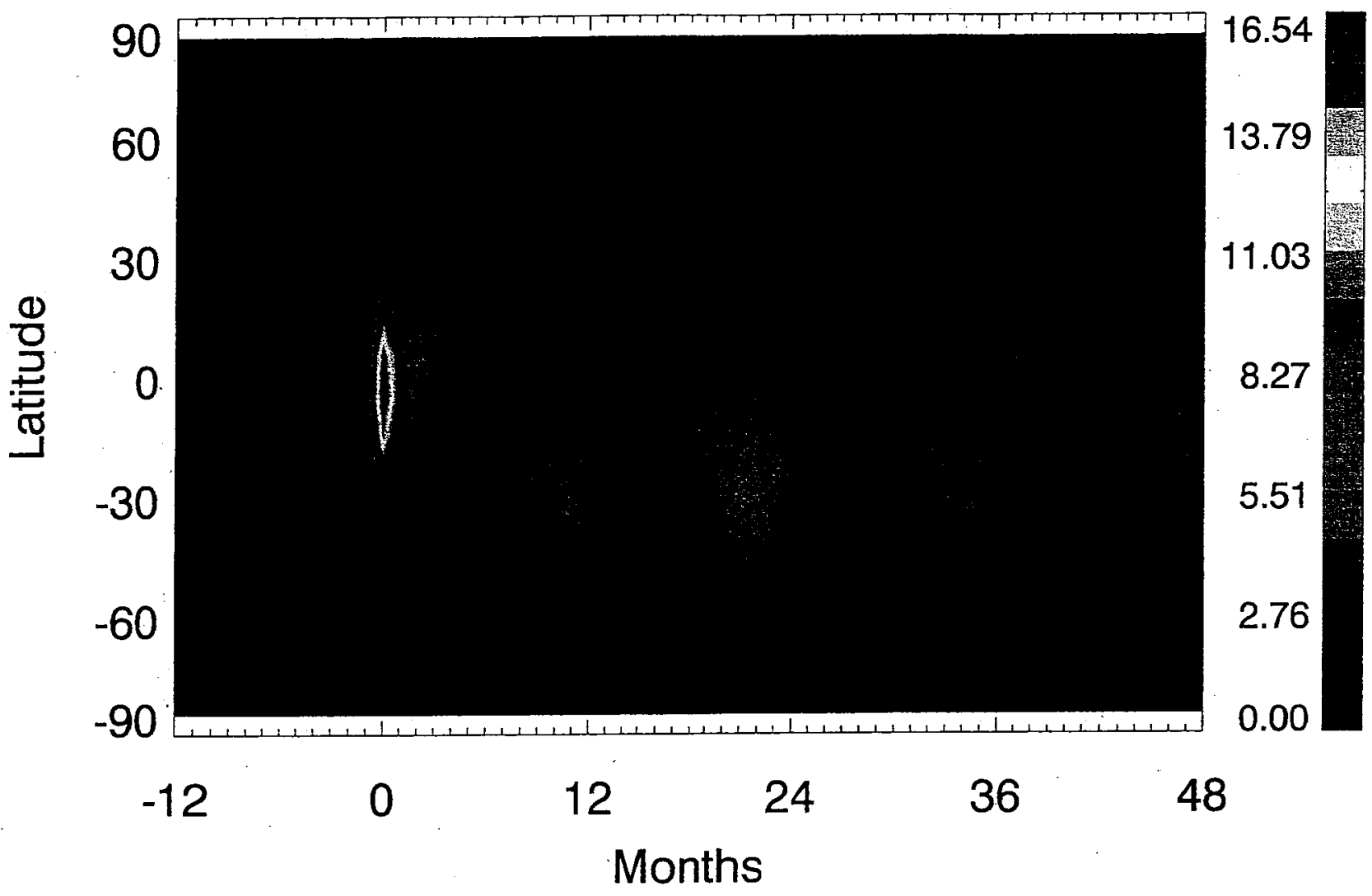

Fig. 3.- Relative DNA damage (dimensionless), normalized by the annual global average damage in the absence of a GRB. (Plotted from one year before the burst to four years after.) 


\title{
Terrestrial Ozone Depletion Due to a Milky Way Gamma-Ray Burst
}

\author{
Brian C. Thomas, Charles H. Jackman, Adrian L. Melott, Claude M. Laird, Richard S. \\ Stolarski, Neil Gehrels, John K. Cannizzo, and Daniel P. Hogan
}

\section{Brief, Popular Summary of the Paper:}

This paper concerns the possibility that a very large gamma-ray burst (GRB) in our Galaxy influenced the Earth's atmosphere in the past billion years. The GSFC two-dimensional (latitude vs. altitude) model was used in this study to investigate this scenario.

A very large ten second burst of gamma and X-rays was simulated to impact the Earth's atmosphere. This radiation produced huge amounts nitrogen-containing compounds, over an order of magnitude larger than background amounts. These nitrogen-containing compounds (primarily nitric oxide and nitrogen dioxide) led to huge amounts of ozone destruction in the stratosphere. Globally averaged total ozone depletion was computed to be a maximum of about $35 \%$. Since the nitrogen-containing compounds can have a fairly long lifetime, significant global ozone depletion $(>10 \%)$ was computed to last for over 5 years past the GRB.

An associated enhancement in ultraviolet (UV) radiation was computed to be one result of the reduced ozone. These enhanced levels of UV can harm various organisms possibly leading to extinction of some species. This study thus suggests that a very large GRB in the Earth's past may have led to a mass extinction event. 